\title{
Pain catastrophizing: a critical review
}

\author{
Phillip J Quartana, PhD, \\ Department of Psychiatry and Behavioral Science, Johns Hopkins University School of Medicine, \\ 600 N Wolfe Street, Baltimore, MD 21287, USA pquarta1@son.jhmi.edu
}

Claudia M Campbell, PhD, and

Department of Psychiatry and Behavioral Science, Johns Hopkins University School of Medicine, 600 N Wolfe Street, Baltimore, MD 21287, USA ccampb41@son.jhmi.edu

Robert R Edwards, PhD ${ }^{\dagger}$

Pain Management Center, Brigham \& Woman's Hospital, 850 Boylston Street, Suite 302, Chestnut Hill, MA 02467, USA Tel.: +16177329486 Fax:+16177329050 rredwards@partners.org

\begin{abstract}
Pain catastrophizing is conceptualized as a negative cognitive-affective response to anticipated or actual pain and has been associated with a number of important pain-related outcomes. In the present review, we first focus our efforts on the conceptualization of pain catastrophizing, highlighting its conceptual history and potential problem areas. We then focus our discussion on a number of theoretical mechanisms of action: appraisal theory, attention bias/information processing, communal coping, CNS pain processing mechanisms, psychophysiological pathways and neural pathways. We then offer evidence to suggest that pain catastrophizing represents an important process factor in pain treatment. We conclude by offering what we believe represents an integrated heuristic model for use by researchers over the next 5 years; a model we believe will advance the field most expediently.
\end{abstract}

\section{Keywords}

attention bias; chronic pain; communal coping; fMRI; helplessness; pain catastrophizing; painrelated surgery; pain sensitivity; primary/secondary appraisal

Pain as a symptom - now considered the fifth vital sign - accounts for approximately $80 \%$ of physician visits and an estimated US $\$ 100$ billion annually between healthcare expenditures and lost productivity [1]. Hence, understanding more definitively the correlates and determinates of pain and suffering is of central importance.

Cartesian notions of pain - a one-to-one relation between tissue damage and the pain experience - dominated the scientific literature for hundreds of years. However, this purely sensory account of pain was inconsistent with observations of great variability in pain reports both in the presence and absence of verifiable physiologic insult. To reconcile these inconsistencies, Melzack and Wall advanced the gate control theory, which conceptualized pain as a complex blend of sensory, emotional, cognitive-evaluative, interpersonal and cultural factors [2].

Psychosocial factors have since become increasingly recognized as important moderators and determinants of the pain experience. One such factor that has garnered great empirical attention is pain catastrophizing.

$\dagger$ Author for correspondence Pain Management Center, Brigham \& Woman’s Hospital, 850 Boylston Street, Suite 302, Chestnut Hill, MA 02467, USA, Tel.: +1 617732 9486, Fax: +1 617732 9050, E-mail: rredwards@ partners.org.

For reprint orders, please contact reprints@expert-reviews.com 
In this updated and comprehensive review of the pain catastrophizing research literature, we critically evaluate the pain catastrophizing construct, positioning catastrophizing in its historical context and detailing a number of unresolved, yet critical, conceptual and measurement issues. We then briefly describe relationships between pain catastrophizing and pain-related outcomes in a variety of samples. Subsequently, we explicate the dominant theories of pain catastrophizing and evaluate the current empirical status of each, then follow with a concise overview of the importance of pain catastrophizing in the context of pain treatment. Finally, we outline a 5-year view that highlights crucial next steps for research, presenting a novel heuristic integrative framework that acknowledges interdependence between the various leading theoretical constructs of pain catastrophizing.

\section{Pain catastrophizing: conceptual \& measurement considerations}

The term catastrophizing was formally introduced by Albert Ellis [3] and subsequently adapted by Aaron Beck [4] to describe a mal-adaptive cognitive style employed by patients with anxiety and depressive disorders. At the core of their definitions of catastrophizing was the concept of an irrationally negative fore-cast of future events. Similarly, pain-related catastrophizing is broadly conceived as a set of exaggerated and negative cognitive and emotional schema brought to bear during actual or anticipated painful stimulation. In early studies, Chaves and Brown defined catastrophizing as a tendency to magnify or exaggerate the threat value or seriousness of pain sensations [5], and Spanos and colleagues emphasized pain-related worry and fear, coupled with an inability to divert attention away from pain [6]. One important limitation of these early studies, however, was the use of nonstandardized interview methods to identify pain catastrophizing, which at least temporarily served as a serious impediment to its measurement.

Other researchers focused on the development of psychometrically sound self-report instruments that could be readily and reliably used with a variety of populations. The Coping strategies Questionnaire (CSQ), developed by Rosentiel and Keefe [7], included a six-item subscale tapping dimensions of helplessness and pessimism in the context of pain. Sullivan et al. elaborated on the CSQ by developing the Pain Catastrophizing Scale (PCS) [8], which incorporates items explicitly designed to assess other elements of catastrophizing. Initial factor analytic work indicated that the PCS yielded three second-order factors (i.e., helplessness, rumination and magnification). Figure 1 presents a graphical representation of the second-order three-factor structure of the PCS and the items comprising each factor. A number of studies have replicated this factor structure using confirmatory factor analytic methods in healthy, pain-free adults [9,10], chronic pain patients [10], across different age groups [11,12] and across diverse cultural groups, and in non-English languages [12-17]. Moreover, the factor structure of the PCS appears to be invariant across sexes and across patient versus nonpatient status $[10,18]$. An area of assessment that is currently underdeveloped is that considering the behavioral elements of pain catastrophizing. To date, there exists no manner of systematically assessing pain catastrophizing behaviors. Assessment of the behavioral dimensions of the catastrophizing construct would likely have important implications for more clearly characterizing the interpersonal consequences and determinants of catastrophizing.

Collectively, pain catastrophizing is characterized by the tendency to magnify the threat value of pain stimulus and to feel helpless in the context of pain, and by a relative inability to inhibit pain-related thoughts in anticipation of, during or following a painful encounter. The assessment of catastrophizing is typically obtained using the CSQ or the PCS. For each, participants are instructed to rate the extent to which they experience each item by recalling former experiences with pain. The PCS assesses three domains believed to comprise much of the pain catatastrophizing construct, while the CSQ only evaluates the helplessness dimension. Hence, the PCS can be considered a broader assessment of the catastrophizing construct than 
the CSQ. Where appropriate, we discuss possible limitations or advantages of using one or the other measure.

\section{Trait (dispositional) versus state (situational) assessment}

Although pain catastrophizing has most typically been conceptualized and assessed as a traitlike or dispositional variable, a handful of recent investigations have assessed pain catastrophizing in a state-like, situation-specific manner. Specifically, some studies have assessed catastrophizing in response to a specific painful encounter, such as laboratory-based noxious stimulation. This mode of assessing catastrophizing is in part theory driven and in part data driven. Regarding the former, initial conceptualizations of catastrophizing considered maladaptive thoughts to be latent and in need of a cue to become manifest [4]. Hence, trait measures of pain catastrophizing (e.g., the CSQ or PCS) may not adequately capture variance in pain report because the referent event is too distal to the moment of measurement. It has been argued that assessment of catastrophizing cognitions in response to a specific stimulus might account for greater variance in pain report than trait measures of catastrophizing. Indeed, some initial investigations supported this prediction, with measures of state pain catastrophizing (i.e., asking participants the extent to which they catastrophized during the painful event that just occurred) showing more robust correlations with pain (Campbell CM et al., Manuscript Submitted) [19-21]. However, a more recent study demonstrated that state pain catastrophizing was not related to pain threshold, pain tolerance or ratings of cold pain [22].

There are a number of shortcomings associated with the state pain catastrophizing literature that are in desperate need of empirical attention. First, the validity and reliability of state pain catastrophizing measures has not been adequately established. The items included in our state pain catastrophizing measure tap only the helplessness and rumination dimensions of catastrophizing [21]. Moreover, for the purposes of this review, exploratory and confirmatory factor analyses were conducted on our state catastrophizing measure, and yielded a one-factor solution in each case. Second, correlations between state and trait measures of pain catastrophizing have ranged from small to moderate in magnitude [Campbell CM et al., Manuscript Submitted; 19-22]. This pattern of relationships is somewhat troubling given that each measure is designed to assess the same underlying cognitive processes. Third, because state pain catastrophizing is assessed following painful stimulation, it is difficult to determine whether the stronger magnitude of relationship for state versus trait pain catastrophizing is not attributable in large part to confounding with the pain experience itself. Subsequent studies will need to carefully determine whether state pain catastrophizing is a construct apart from pain and its affective constituent. Lastly, although the argument has been thoughtfully advanced that pain catastrophizing might represent a type of latent construct, requiring sufficient activation to exert its effects (Campbell CM et al., Manuscript Submitted) [23], support for this argument will need to be derived from moderational analyses that examine whether trait pain catastrophizing is related most strongly to pain outcomes only when the pain stimulus or painful event elicits a catastrophizing response (i.e., state catastrophizing). To our knowledge, such an analysis has not been conducted.

\section{Construct redundancy}

Pain catastrophizing is but one of a number of variables that tap into a negative pain schema, including pain anxiety, fear of pain and pain helplessness. Moreover, pain catastrophizing shares significant variance with broader negative affect constructs, such as depression, anxiety, anxiety sensitivity, worry and neuroticism [24-27]. A handful of recent studies call into question the extent to which pain catastrophizing is conceptually distinct from these other measures. 
It is commonplace to statistically control for depressive symptoms when investigating relations between pain catastrophizing and pain-related outcomes. Nevertheless, it must be kept in mind that catastrophizing shares elements in common not only with depression, but also with anxiety and its associated disorders, and perhaps anger and hostility as well. A recent investigation attempted to examine whether pain catastrophizing (assessed with the CSQ) is distinct from other constructs that tap negative affectivity in general, and negative cognitive-affective processes tied to pain more specifically [26]. Their results suggested that pain catastrophizing was strongly associated with a number of indices of negative mood, and accounted for minimal variance in pain outcomes above and beyond negative mood. These findings raise the possibility that pain catastrophizing is associated with exaggerated pain by way of negative affective states and/or dispositions, and suggest that additional studies should be conducted to continue to examine the uniqueness of the catastrophizing construct.

Another recent study indicated high correlations between fear of pain and pain catastrophizing $(r=0.45)$, and indicated that when both variables were considered simultaneously in regression models, only fear of pain predicted cold pressor pain threshold and intensity [28]. Sullivan and colleagues found a somewhat different pattern of findings in an experimental study among healthy pain-free participants, and in a clinical study among patients with pain [29]. Strong relationships were noted between fear of pain, catastrophizing, and state and trait anxiety; however, only pain catastrophizing predicted pain intensity. Interestingly, only the Sullivan et al. study employed the PCS [29], whereas the other two studies employed the CSQ to assess catastrophizing, making direct comparisons across studies difficult [26,28].

In summary, there are some conflicting evidence as to the uniqueness of the pain catastrophizing construct above and beyond negative affectivity in general, or with regard to other negative pain-related cognitive process variables, such as fear of pain. Nonetheless, substantial empirical evidence highlights the importance of pain catastrophizing in shaping the experience of acute and chronic pain.

\section{Association of pain catastrophizing with pain-related outcomes}

Pain catastrophizing has been associated with a number of indices of pain sensitivity in the context of experimental pain testing paradigms, both among healthy, pain-free participants and individuals with various chronic pain conditions [30,31]. The literature also points to consistent and generally robust associations observed between pain catastrophizing and an array of clinical pain-related outcomes, including measures of clinical pain severity, pain-related activity interference, disability, depression (and other negative mood indices) and alterations in social support networks [30,31]. Moreover, catastrophizing has been linked to increased behavioral expressions of pain, as well as a variety of illness behaviors (e.g., more frequent visits to healthcare professionals). It is important to note that the magnitude of these relationships is variable, with catastrophizing accounting for minimal variance in pain severity in some studies, and up to $31 \%$ of the variance in pain severity in others [30].

Importantly, pain catastrophizing is also related prospectively to adverse pain-related outcomes $[30,32,33]$. Indeed, pain catastrophizing-assessed presurgery has been shown to account for significant variance in postsurgical pain ratings, narcotic usage, depression, pain-related activity interference and disability levels [34-41]. As indicated earlier, pain catastrophizing has been cross-sectionally and prospectively linked to exaggerated negative mood and depression. This is perhaps a critical observation, given that chronic pain is associated with high rates of suicide [42]; indeed, a study by Edwards et al. suggested that pain catastrophizing was related to increased suicidal ideation in a large sample of chronic pain patients [43].

In summary, pain catastrophizing has emerged as a rather potent predictor of a variety of painrelated outcomes, both in pain-free and chronic pain patient samples. We next address some 
of the postulated mechanisms by which pain catastrophizing might be associated with adverse outcomes (Table 1).

\section{Theoretical mechanisms of action \& supporting evidence}

\section{Appraisal theory}

Some have framed pain catastrophizing in the context of Lazaraus and Folkman's transactional model of stress and coping [44], at the core of which are the notions of primary and secondary appraisals. These appraisal processes act in a transactional manner to shape the cognitive and behavioral coping strategies that are ultimately deployed. Primary appraisal concerns judgments regarding whether a potential stressor is irrelevant, benign-positive or stressfulnegative. Secondary appraisals are beliefs about coping options and the extent to which they will be successful or not. According to Severijns and colleagues, magnification and rumination may reflect a focus on and evaluation of a painful stimulus as extremely threatening (primary appraisal), whereas helplessness may reflect secondary appraisals of inability to cope [45]. Whether these components of catastrophizing work interactively to predict behavior has not been examined in a systematic fashion, but certainly would represent an exciting avenue for future research. Even so, there are threads of evidence suggesting that catastrophizing is associated with other appraisal processes, such as self-efficacy concerning one's ability to control their pain [30].

\section{Attention bias/information processing}

Some researchers have proposed that pain catastrophizing might be characterized by attention and information processing biases analogous to those observed in individuals with anxiety and depressive disorders [46]. Specifically, it has been argued that pain catastrophizing amplifies the experience of pain via exaggerated attention biases to sensory and affective pain information. Indeed, pain catastrophizing is rooted in traditional cognitive-behavioral conceptualizations of anxiety and depression, and is characterized substantially by a relative inability to suppress or inhibit pain-related cognitions. To date, numerous laboratory-based attentional, information-processing and classical conditioning paradigms have provided empirical support for attention bias as a key cognitive mechanism underlying adverse outcomes associated with pain catastrophizing.

Even in noncatastrophizers, pain demands attention, interrupts ongoing activity and interferes with mental processes [46,47]. These attentional and behavioral consequences of pain are largely adaptive, given that pain signals threat. However, in persons who tend to catastrophize, pain might demand attention to the point of cognitive and behavioral immobilization.

Van Damme and colleagues used a cueing paradigm to determine whether pain catastrophizers evince exaggerated attentional engagement, retarded attentional disengagement or both, to pain $[48,49]$. Their data indicated that pain catastrophizing was associated with retarded disengagement from both pain cues and painful stimuli, suggesting that either the threat of pain or pain itself may engage negative pain schemas most readily among high pain catastrophizers, similar to processes that occur in patients with phobias or depressive disorders. Interestingly, their findings also suggested that pain catastrophizers were relatively unable to utilize disconfirming evidence that pain cues were not necessarily followed by pain experiences, indicating that those high in pain catastrophizing may overestimate the probability of experiencing pain. These data dovetail with clinical findings suggesting that graded exposure to movement in chronic low back pain is sometimes inefficacious for pain catastrophizers [50], perhaps because they are unable to assimilate evidence that pain does not necessarily follow movement. 
The aforementioned studies suffered from a lack of concurrent administration of measures tapping similar pain-related and unrelated negative mental sets that also might be related to exaggerated attentional interference. For example, fear of pain has been linked to an attentional bias for pain-relevant stimuli using modified Stroop [51], dot-probe [52] and attentional interference [47] paradigms. Vancleef and Peters examined performance on an auditory discrimination task during electrocutaneous stimulation, and correlated performance with measures of pain catastrophizing, anxiety sensitivity and injury/illness sensitivity [53]. While the three measures were highly intercorrelated, with $\mathrm{r}$ values ranging from 0.49 to 0.67 , only pain catastrophizing was correlated with attentional interference, defined as performance decrements on the auditory discrimination task during electrocutaneous simulation. By contrast, a recent study by Vangronsveld et al. using a dual task paradigm found that neither pain catastrophizing scores nor other conceptually relevant variables were associated with attention interference in neck pain patients or controls [54]. However, a restricted range of catastrophizing scores in their sample may have obscured relations between catastrophizing and attentional interference. Using a structural equations approach, Goubert $e t$ al. found that neuroticism - a measure of negative affectivity - was related to pain vigilance through its association with pain catastrophizing [55].

It is also possible to manipulate attentional processes, as in a study by Michael and Burns, who examined the effects of different 'styles' of processing information on relationships between pain catastrophizing and cold pain sensitivity [56]. Following an initial 'typical' cold pressor task, during a second cold pressor, participants read aloud a list of control words (e.g., desk), pain-sensory words (e.g., burning) or pain-affect words (e.g., irritating). Given that catastrophizers may exaggerate the negative affective elements of pain via their negative and affectively-tinged mental set, it was expected that reading affective stimulus words would lead to increases in pain sensitivity proportional to the degree of catastrophizing. Indeed, in the affect focus condition, higher levels of pain catastrophizing were associated with decreased pain threshold and tolerance from the first to the second cold pressor. This relationship was not observed in control and sensory focus conditions.

In summary, preliminary studies using diverse experimental methods have suggested that pain catastrophizing is associated with a heightened attentional bias to pain-relevant stimuli (perhaps its negative affective element in particular) and an inability to disengage from pain or pain cues. However, none of these studies has examined systematically whether catastrophizing is associated with an attention bias distinct from other constructs denoting painrelated negative mental sets, such as fear of pain and pain anxiety. Moreover, only one of the aforementioned studies controlled for depression in analyses. It will be crucial to determine whether pain catastrophizing is associated with attention bias to pain-related stimuli above and beyond mere negative emotionality.

\section{Catastrophizing in a communal context}

Sullivan and colleagues advanced a communal coping model, suggesting that catastrophizing represents an interpersonal style of coping with pain and suffering [30]. The model hinges on the notion that catastrophizing represents a behavioral coping strategy employed by individuals experiencing pain to elicit emotional and/or tangible support from others, thereby positively reinforcing pain and illness behaviors and undermining successful adaptation to pain.

A number of studies have provided support for the communal coping model. In a sample of patients with gastrointestinal cancer pain, Keefe and colleagues demonstrated that patient catastrophizing was associated with greater receipt of instrumental support from the partner [57]. Moreover, partners of patients high in catastrophizing perceived the patient to be in greater pain. Another study demonstrated that pain catastrophizing was associated with both solicitousness and pain severity, and the association between pain and catastrophizing was 
most robust for those who resided with a spouse or partner [58]. Pain catastrophizing has also been associated with higher observer ratings of pain behaviors during acute cold pain [59], suggesting that catastrophizers may engage in expressive pain behaviors to solicit supportive responses from others.

Pain catastrophizing has also been associated with exaggerated punishing and critical responses from spouses and partners [57,60,61]. Moreover, punishing responses have been shown to account for the relationship between pain catastrophizing and pain-related disability and depressed mood [61], suggesting that expressions of pain elicited by pain catastrophizers, although aimed at soliciting supportive responses and relational proximity, may actually lead to relationship strain and negative interpersonal responses. An interesting moderation analysis by Cano helped to reconcile these seemingly divergent interpersonal consequences of pain catastrophizing [62]. Specifically, Cano's analysis suggested that pain catastrophizing is associated with supportive response among patients with short pain duration, but punishing/ negative responses among those with longer pain duration. A similar pattern of relations was also noted by Buenaver and colleagues [61].

In addition, a study by Lackner and Gurtman, using a circumplex model of interpersonal behavior, showed that pain catastrophizing is associated with greater interpersonal problems involving friendly and submissive interactions (believed to indicate a high need and demand for support) [63]. The relation between interpersonal problems and pain catastrophizing remained significant even after statistically controlling for general symptomatic distress. These data suggest that pain catastrophizing is related to an interpersonal style characterized by submissiveness and high levels of dependency and support seeking. It was more recently shown that pain catastrophizing is associated with an insecure adult attachment style [64]. More specifically, pain catastrophizing was linked to beliefs that others will not provide support in times of distress. Pain catastrophizing, as well as fear of pain and hypervigilance to pain, were associated with perceptions of the self as incapable of coping with distress. These findings suggest that both self and other aspects of insecure attachment are related to catastrophizing.

\section{CNS mechanisms}

A handful of studies have examined whether pain catastrophizing is associated with alterations in endogenous pain modulation pathways. Indeed, Sullivan et al. postulated that the cognitiveaffective processes that comprise pain catastrophizing might amplify the experience of pain by increasing nociceptive transmission via spinal gating mechanisms [30]. However, several studies of the nociceptive flexion reflex, a polysynaptic spinal reflex that subserves withdrawal from potentially noxious stimuli, suggest that pain catastrophizing is not associated with nociceptive flexion reflex threshold [65]. By contrast, pain catastrophizing is associated with alterations in supraspinal endogenous pain-inhibitory and -facilitatory processes. Diffuse noxious inhibitory controls (DNICs) are a commonly used measure of endogenous descending pain inhibition. DNIC is a phenomenon in which the activity of pain-signaling neurons in the dorsal horn is attenuated in response to noxious stimuli applied to a remote site of the body. It is assessed by ascertaining pain ratings for a 'test' stimulus before and after application of a 'conditioning' stimulus. DNIC is quantified as a reduction in pain in the test stimulus over repeated trials with the conditioning stimulus. Recently, Weissman-Fogel et al. identified negative relationships between pain catastrophizing and DNIC, although these authors did not assess measures of negative affect or fear of pain [66]. Temporal summation or 'wind-up' is a commonly employed measure of pain facilitation, and it has been conceptualized as a psychophysical marker of central sensitization-like processes [67]. Temporal summation is assessed by administering repetitive, identical, phasic noxious stimuli. Typically, pain ratings increase across successive stimuli, reflecting a 'summation' process. Temporal summation is quantified as the difference between the highest-rated stimulus and the initial stimulus of a 
given sequence. A handful of studies using repetitive thermal stimulation have revealed correlations between pain catastrophizing and the degree of temporal summation [68-70].

These findings suggest that pain catastrophizing might be associated with diminished endogenous inhibition of pain coupled with central sensitization, which may represent a CNS mechanism by which pain catastrophizing is associated with the development, maintenance and aggravation of persistent pain. The causal nature of these relationships remains to be determined, and will be an important area for future study. For instance, it is plausible that altered CNS mechanisms cause an individual to catastrophize about pain. Moreover, it will be crucial to determine whether relations between altered CNS pain modulation pathways are uniquely associated with pain catastrophizing, or whether it is held in common with negative affect or other negative pain-related cognitions.

\section{Physiological pathways}

Although investigation of the physiological consequences of catastrophizing is a relatively new area of study, some interesting data have surfaced that suggest that pain catastrophizing might be related to altered physiological responses to stress and pain. Aberrant patterns of muscle tension have received some initial investigation as potential meditational pathways [71,72]. Based on Flor's [73,74] notions of 'symptom-specificity,' it has been proposed that pain catastrophizing might be related to greater chronic low back pain severity and disability via specifically exaggerated muscular responses near the site of injury. Indeed, Quartana et al. showed that pain catastrophizing was positively correlated with lower paraspinal, but not trapezius muscle responses, to cold pain in a sample of chronic low back pain patients [71]. This relationship remained significant even after controlling for symptoms of depression. The relationship between catastrophizing and lower paraspinal or symptom-specific muscle responses to pain was augmented by efforts to suppress thoughts of pain. An interactive effect of pain catastrophizing and resting lower paraspinal muscle activity on clinical pain severity was also recently observed, such that pain catastrophizing was associated with greater clinical pain severity ratings most robustly for those with higher resting lower paraspinal muscle activity [72].

Some data also suggest that pain catastrophizing is associated with altered hypothalamicpituitary-adrenal axis activity. In a sample of spinal pain patients scheduled for lumbar disc surgery, pain catastrophizing (in addition to other pain-related negative cognitive variables) was associated with less variability in diurnal cortisol activity [75]. In addition, a recent study by our group showed that pain catastrophizing (after controlling for general symptom distress and depression) was associated with greater interruption of the morning cortisol decline in response to a battery of experimental pain testing in samples of both pain-free subjects and patients with persistent orofacial pain [76]. Although these data suggest that pain catastrophizing might be associated with aberrant hypothalamic-pituitary-adrenal axis activity, the functional significance and robustness of these findings requires further study.

The arthritis literature has identified helplessness as a potentially important factor in shaping some measures of disease activity [31], suggesting the possibility that pain catastrophizing might be related to altered neuroimmunologic responses to pain. Our group has recently shown positive correlations between state pain catastrophizing and IL-6 responses to experimental pain testing among healthy, pain-free participants (Quartana PJ et al., Manuscript Submitted). Although the possibility remains that pain catastrophizing influences pain via immunologic pathways, this area is need of further empirical study. Lastly, there is some initial data suggesting genetic factors associated with high pain sensitivity and development of temporomandibular disorder (TMD) pain (e.g., catechol- $O$-methyltransferase $[77,78]$ ), may act in a synergistic manner with pain catastrophizing to affect clinical pain severity $[79,80]$. 


\section{Neural correlates}

The advent of noninvasive brain imaging techniques has made possible the identification of various top-down and bottom-up neural circuits involved in the experience of pain, and hundreds of functional MRI studies have examined the brain's processing of pain-related information [81]. Over the past few years, several studies have also assessed the neural correlates of pain catastrophizing using functional neuroimaging during application of standardized pain stimuli. Given that pain catastrophizing is associated with exaggerated negative affective responses to pain, as well as maladaptive cognitive modulation of pain, investigators have focused particularly on those brain regions involved in processing and regulation of the unpleasantness dimension of pain [82], and emotion more broadly [83], such as the anterior cingulate cortex (ACC), and the dorsolateral and ventromedial prefrontal cortex.

Seminowicz and Davis found that during mild and intense pain, pain catastrophizing was not significantly associated with activation in primary or secondary somatosensory cortices [84]. During mild pain, pain catastrophizing was associated with exaggerated activity in the PFC, insular cortex and caudal ACC, suggesting exaggerated processing of the affective dimension of pain in particular. During more intense pain a somewhat different pattern emerged: pain catastrophizing was negatively associated with caudal ACC and insular cortex activity, possibly suggesting failure of activation of top-down inhibitory control in response to severe pain. Importantly, these associations were independent of neuroticism, a measure of dispositional negative affectivity that is highly correlated with pain catastrophizing.

A recent study showed a similar pattern of correlations using functional neuroimaging with painful and nonpainful thermal stimuli, but with measures of pain-related fear and anxiety sensitivity (a proxy for generalized anxiety or negative affect) and not pain catastrophizing per se [85]. The authors found that fear of pain correlated positively with pain-related activation in the ventral lateral PFC - believed to be involved in affect regulation - and the posterior ACC - believed to be involved in monitoring and evaluation of affective states in the context of stress and pain. This pattern of relations was not accounted for by generalized anxiety. These data suggest that fear of pain and pain catastrophizing are likely to activate highly overlapping neural circuits. Finally, in a study of fibromyalgia patients, Gracely and colleagues found that pain catastrophizing (after controlling for depression) was associated with activation of the dorsolateral prefrontal cortex, dorsal ACC, medial PFC and motor areas [86].

These data suggest that pain catastrophizing, in both healthy volunteers and pain patients, is related to greater activity in areas implicated in affective processing of pain, attention to pain, and perhaps with premotor and motor regions associated pain behaviors. It is important to note, however, that these brain regions are broadly implicated in a variety of emotional and cognitive processes. Again, the uniqueness of these relations requires further assessment via studies that include not only a measure of catastrophizing, but other pain-related negative cognitive variables, such as fear of pain and pain anxiety.

\section{Pain catastrophizing as a process variable in pain treatment outcomes}

Given pain catastrophizing's consistent relations with pain-related outcomes, as well as with neural, physiological, cognitive, affective and interpersonal factors associated with pain and suffering, it follows that reductions in pain catastrophizing should be related to reductions in pain, disability and interpersonal discord among persons with persistent pain. Indeed, a core component of multidisciplinary pain treatment is often cognitive-behavioral techniques involving reducing catastrophizing and enhancing adaptive pain-coping skills. The use of such techniques is based on the well-documented notion that the manner in which a patient assesses their pain and contextual surroundings has a profound influence on pain, disability and emotional wellbeing [87]. 
A number of studies have examined whether pain catastrophizing indeed is an active cognitive process variable in multidisciplinary pain treatment settings, and have shown that pre- to posttreatment reductions in cognitive process factors, including but not limited to pain catastrophizing, are associated with pre- to post-treatment reductions in pain severity [8890]. Concerning pain catastrophizing specifically, Jensen et al. found that decreases in pain catastrophizing were associated with 6- and 12-month improvements in disability, pain intensity and depression in a heterogeneous sample of pain patients [91]. A more recent investigation by Jensen et al. found that increases in depression and disability from immediately post-treatment to 12-months post-treatment were associated concurrently with changes in pain beliefs and catastrophizing [92]. These data indicate that multidisciplinary pain treatment programs, of which cognitive-behavioral intervention is a core ingredient, can lead to reductions in pain catastrophizing concurrent with reductions in pain, disability and depression. Moreover, they highlight the fact that following treatment termination pain catastrophizing increases concurrently with depression and disability levels.

Although supportive of the idea that multidisciplinary pain treatment programs can decrease pain, disability and depression by way of reduction in pain catatastrophizing, the aforementioned studies were based on correlations between changes in cognitive process factors (e.g., pain catastrophizing) at one point in time with pain-related outcomes at the same point in time. Hence, it is not possible to conclude that reductions in pain catastrophizing preceded improvement in pain, disability and depression. Interestingly, results from an initial investigation in a heterogeneous pain population (predominantly low back pain) using a crosslagged panel design and analysis [93] indicated that pre- to mid-treatment changes in pain catastrophizing and pain helplessness predicted mid- to late-treatment changes in pain severity and pain-related interference, but not vice versa [94]. However, when depression was statistically controlled, only early treatment changes in pain helplessness predicted late treatment changes in pain outcomes, suggesting that early reductions in the helplessness dimension of catastrophizing might be most critical for later changes in pain-related outcomes. A follow-up study, however, showed that the lion's share of late treatment changes in pain outcomes were attributable to variance shared in common with early treatment changes in pain helplessness, pain anxiety and pain catastrophizing; that is, no one cognitive process factor emerged as a unique predictor of pain outcomes [95]. Moreover, analyses of magnitude of changes suggested that early treatment changes in cognitive process factors did not predict extensive (i.e., $>1$ standard deviation) late treatment improvements in pain. Nonetheless, these data suggest that changes in cognitive process variables, such as pain catastrophizing, precede changes in pain-related outcomes in the context of multidisciplinary pain treatment programs.

There has been a recent increase in the number of studies of pain catastrophizing as a process factor in clinical trials of stand-alone cognitive-behavioral therapy for pain-related disorders (e.g., TMD), as well as in the context of exposure-based therapy. Turner et al. observed significantly greater improvements in pain catastrophizing assessed with the CSQ and PCS among TMD patients that received pain management training (brief cognitive-behavioral therapy) compared with standard self-management care using both self-report and daily diary assessment methods [96,97]. A subsequent analysis of those data revealed that changes in pain catastrophizing (as assessed with the CSQ), self-efficacy and pain beliefs (e.g., control over pain) mediated treatment-related changes in pain intensity and pain-related interference [98]. However, further analyses indicated that change in perceived control of pain - and not pain catastrophizing - accounted for the most variance in outcomes (65 vs 29\%, respectively). Interestingly, changes in the rumination subscale of the PCS did not account for significant variance in outcomes.

In a very recent study of subacute low back pain patients that compared graded exposure/ activity plus basic physical therapy to traditional physical therapy, changes in pain intensity 
were associated with changes in pain catastrophizing at 6 months post-treatment, irrespective of treatment condition [99]. Similar findings were evident in a study comparing cognitivebehavioral therapy, an exercise-based behavioral intervention, and their combination in patients with chronic low back pain [100]. Even in the purely 'behavioral' treatment arm (i.e., regular aerobic exercise), reductions in pain catastrophizing at least partially mediated the reductions in disability and pain intensity achieved by the intervention. These data suggest that behavioral interventions absent of any explicit cognitive intervention are associated with concurrent changes in catastrophizing and pain intensity. The George et al. study also revealed that 6-month reductions in pain were associated with reductions in fear-avoidance beliefs [99]. Unique versus common contributions of these cognitive process variables were not examined. These data are consistent with dismantling studies conducted in the context of depression and anxiety disorders (of which catastrophizing is a core cognitive style), which have shown similar magnitude of immediate and long-term therapeutic effects of purely behavioral interventions versus cognitive plus behavioral interventions [101].

Lastly, Quartana et al. employed cross-lagged panel analyses in a sample of musculoskeletal pain patients and found that pain helplessness (a dimension of pain catastrophizing) preceded transition from a precontemplative stance to a 'self-management' approach to pain symptoms, even controlling for early-and late-treatment changes in depressed affect [102]. Hence, early treatment changes in maladaptive pain schemas may need to take place before patients adopt adaptive self-management strategies, which are robustly related to improvements in pain and disability (Quartana PJ et al., Manuscript in Preparation) [103,104].

Taken together, these data suggest that treatment-associated reductions in pain catastrophizing, perhaps the helplessness dimension in particular, are associated with improvements in pain intensity, pain-related activity interference and disability. However, the specificity of this effect remains questionable. Specifically, whether changes in catastrophizing need to occur to yield beneficial outcomes, or whether change in other cognitive process factors (e.g., fear of pain) yields similar benefit is not fully understood and is in need of further study.

\section{Expert commentary}

The catastrophizing literature to date provides rather demonstrative evidence for the influence of catastrophizing in shaping emotional, functional and physiological responses to pain. At this point, in our view, enough evidence has accumulated to suggest that high levels of catastrophizing about pain should be considered a 'risk marker' for adverse immediate and long-term pain-related outcomes. Moreover, the apparent role of catastrophizing in contributing to the effectiveness of a variety of pain treatments, from multidisciplinary interventions to cognitive-behavioral therapy to exercise treatments (e.g., [99,100]), suggests that it may become a key target of many chronic pain management programs.

In order to advance the field of catastrophizing research, increasing the focus on several key areas will be important. First, assessment issues represent a barrier to furthering our understanding of this construct; catastrophizing has been assessed using global, retrospective self-report (e.g., the PCS), using situation-specific recall of catastrophizing cognitions during a recently-experienced, standardized pain episode and using diary assessment of moment-tomoment cognitions in daily life. In general, the associations between these measures of catastrophizing are modest, and it will be crucial to characterize the determinants and consequences of catastrophizing in particular contexts. Moreover, it is important to bear in mind that each of these assessment methods tells little, if anything, about the process of catastrophizing. The utilization of these self-report methods merely speaks to a cognitive product. Using paradigms that manipulate intensity of pain and concomitantly assess pain 
catastrophizing (i.e., product) and attentional bias (i.e., process) may shed additional light on the processes that characterize the catastrophizing construct.

Second, few studies comprehensively assess other negative emotional and cognitive processes that overlap (often substantially) with the construct of catastrophizing. It will be critical to place catastrophizing within the context of such other processes as fear of pain, pain-related anxiety, pain self-efficacy and somatization. Perhaps even more critical will be the determination of the uniqueness of the catastrophizing construct; that is, whether it accounts for clinically meaningful variance in a variety of pain-related and interpersonal outcomes above and beyond other purported pain-related cognitive, behavioral and affective process variables.

Third, a handful of mechanisms have been proposed to account for the effects of catastrophizing on outcomes, ranging in scope from the neurophysiological to interpersonal. Mechanistic studies of catastrophizing have predominantly focused on a unitary potential mechanism; however, the hypothesized pathways are not mutually exclusive, and future catastrophizing research would benefit from a more comprehensive consideration of multiple processes.

Finally, we recommend that researchers in the field of pain-related catastrophizing regain sight of the historical roots of catastrophizing. More specifically, the construct stems from a broader notion of catastrophizing in the context of psychiatric disorders such as major depression and generalized anxiety disorder. Although some have utilized methodologies and theoretical models advanced by basic psychopathology researchers to better understand the pain catastrophizing construct $[48,49,51,52,105]$, such an application is perhaps occurring at too deliberate a rate. For instance, there is only one published study that attempted a manipulation of pain catastrophizing to determine the construct's causal influence on pain experience [106]. These authors instructed participants to recite catastrophizing items taken directly from the PCS during a painful cold pressor task, and then reassessed catastrophizing following the cold pressor task. Although this method led to modest increases in pain catastrophizing, such changes were not associated with pain. Although this method is certainly face valid, it bears little on the processes purported to underlie pain catastrophizing and it is not possible to rule out the possibility that any changes in catastrophizing were because of experimental demand. Drawing from the psychopathology literature, there are more subtle manipulations available that bear more directly on purported catastrophizing processes and are much less susceptible to response bias and/or experimental demand. For instance, attentional training paradigms are being utilized to induce an anxiety-like information processing bias [107] and, conversely, to eliminate pre-existing information processing biases corresponding to high levels of anxiety $[108,109]$. If catastrophizing is in part characterized by attentional bias directed toward threatening aspects of pain [46-49], then induction of such an attentional bias should lead to catastrophizing processes that in turn enhance subjective pain experience and, perhaps, underlying neurophysiological function. Conversely, investigators might consider training high catastrophizers to attend to less threatening information in the context of pain, in turn exerting salutary effects on responses to acute and clinical pain. In short, we strongly believe that continued application of well-founded paradigms from other literatures will further enhance our ability to characterize the pain catastrophizing construct and the processes by which it impinges upon pain and suffering.

\section{Five-year view}

Speculation regarding the direction of future catastrophizing research could potentially fill an entire review, but we anticipate some critical areas over the course of the next 5 years.

Application of well-validated experimental paradigms from other literatures, such as experimental psychopathology (e.g., [105]), will continue in order to better understand the shared and unique determinants of pain catastrophizing on pain-related outcomes. In addition, 
use of multiple neuroimaging techniques will further characterize the neural underpinnings of catastrophizing, and the role of various neurotransmitter substances. For example, PET and magnetic resonance spectroscopy methodologies can be used to evaluate the activity of particular neurotransmitter systems in the brain under various experimental conditions. There will also be studies of the development and determinants of pain-related catastrophizing. For instance, we have only recently begun to evaluate potential genetic moderators of individual differences in catastrophizing $[79,80]$, or the contribution of early life experiences, such as parenting styles, to the development of catastrophizing among children and adolescents [110].

Assessment issues represent a barrier to furthering our understanding of catastrophizing. Consequently, we expect this field of research to benefit greatly from studies that utilize multicontextual assessment of catastrophizing (e.g., using daily diaries, spouse reports and application of standardized stress/pain tasks to elicit catastrophizing), and that place catastrophizing within the context of other related cognitive processes (e.g., attentional bias) in order to determine unique versus common contributions of catastrophizing to pain-related outcomes.

Finally, and most crucially, we believe that for the field to most readily advance will require explorations of interactive mechanisms involved in pain-related catastrophizing. To that end, we have advanced a heuristic model for the integrative study of pain catastrophizing (Figure 2). This model explicitly notes and emphasizes possible inter-relationships between the aforementioned theoretical mediators and moderators of catastophizing's effects on pain and pain-related outcomes, such as disability and social networks. Importantly, we believe this model can most expediently advance the field by focusing hypotheses on multiple process and/ or moderating variables theoretically and/or conceptually related to catastrophizing. For instance, genetic polymorphisms might contribute to exaggerated inflammation, in turn leading to elevated levels of pain and so lead to a catastrophizing process and, ultimately, to sociallyobservable expressions of pain behaviors. As a second example, family history of chronic pain may lead to exaggerated threat perceptions (i.e., catastrophizing) for a child experiencing a painful episode (e.g., tonsillectomy), thereby leading to increased subjective pain experience and concomitant maladaptive neurophysiological responses. Utilization of a framework such as that illuminated in Figure 2 therefore has the potential to move researchers toward integrative versus piecemeal studies of pain catastrophizing, and in doing so circumvent potentially unhelpful debates over whether catastrophizing is an appraisal, a coping strategy or a maladaptive belief. It is most plausible that catastrophizing is an unfolding process [45] with multiple determinants (e.g., primary and secondary appraisals) and moderators (e.g., genetic polymorphisms, such as catechol- $O$-methyltransferase). We believe the coming years will yield exciting findings concerning not only pain catastrophizing, but other negative painrelated cognitive constructs, such as fear of pain, pain anxiety and pain helplessness. Such findings will no doubt lead to improved, perhaps even novel, therapeutic interventions for those suffering from chronic, often disabling, pain.

\section{Key issues}

- Pain catastrophizing stems from original conceptualizations of catastrophizing in the context of depressive and anxiety disorders advanced by Albert Ellis and Aaron Beck.

- Pain catastrophizing has been associated with a number of pain-related outcomes, including acute and chronic pain severity, altered CNS pain processing (e.g., diminished endogenous pain inhibition), exaggerated healthcare utilization, postsurgical pain outcomes and disability, and pain-related activity interference. 
- Catastrophizers appear to attend most readily to pain-related stimuli and have a particularly pronounced inability to disengage cognitive resources from such stimuli.

- Catastrophizing behaviors appear to exert significant, and potentially deleterious, effects on a patient's support network. For example, longer durations of pain appear to exaggerate the relationship between pain catastrophizing and punishing responses from the spouse/significant other. Development of systematic behavioral assessments of catastrophizing would be timely.

- There are preliminary data suggesting that pain-related catastrophizing is associated with altered hypothalamic pituitary responses to pain and amplified activation in neural regions implicated in processing and regulation of affective components of pain (e.g., anterior cingulate cortex).

- Pain-related catastrophizing appears to represent a robust cognitive process variable in multidisciplinary pain treatment outcomes studies, as well as in cognitive behavioral and stand-alone physical therapy (i.e., behavioral) for chronic pain.

- Future studies will need to more rigorously explore the empirical distinctiveness of the pain catastrophizing construct apart from related constructs, such as fear of pain, as well as from more general constructs imbued with negative affectivity, such as depression, anxiety and neuroticism.

- Innovative assessment techniques that move beyond self-report, or that can augment self-report methods, need to be advanced so that the process of catastrophizing can be more readily characterized.

- Greater reliance on theory and methods employed by experimental psychopathology researchers may shed novel light on the processes that underlie the effects of catastrophizing on pain-related outcomes.

- For the field of pain catastrophizing research to advance will require a concerted effort to employ studies that aim to systematically and concurrently examine multiple proposed processes versus one process or another.

\section{Acknowledgments}

Financial \& competing interests disclosure

Phil Quartana is funded by an NIH Training Grant (T32MH075884), Claudia Campbell is funded by an NIH grant (F32NS063624) and Robert Edwards is also funded by an NIH grant (K23AR051315). The authors have no other relevant affiliations or financial involvement with any organization or entity with a financial interest in or financial conflict with the subject matter or materials discussed in the manuscript apart from those disclosed.

No writing assistance was utilized in the production of this manuscript.

\section{References}

Papers of special note have been highlighted as:

- of interest

•• of considerable interest

1. Gatchel RJ, Peng YB, Peters ML, Fuchs PN, Turk DC. The biopsychosocial approach to chronic pain: scientific advances and future directions. Psychol Bull 2007;133(4):581-624. [PubMed: 17592957] 
2. Melzack R, Wall PD. Pain mechanisms: a new theory. Science 1965;150:971-979. [PubMed: 5320816]

3. Ellis, A. Reason and Emotion in Psychotherapy. Lyle Stuart; NY, USA: 1962.

4. Beck, AT.; Rush, AJ.; Shaw, BF.; Emery, G. Cognitive Therapy of Depression. Guilford Press; NY, USA: 1979.

5. Chaves JF, Brown JM. Spontaneous cognitive strategies for the control of clinical pain and stress. J Behav Med 1987;10:263-276. [PubMed: 3612783]

6. Spanos NP, Radtke-Bodorik HL, Ferguson JD, et al. The effects of hypnotic suggestibility, suggestions for analgesia, and utilization of cognitive strategies on the reduction of pain. J Abnorm Psychol 1979;88:282-292. [PubMed: 500957]

7. Rosentiel AK, Keefe FJ. The use of coping strategies in chronic low back pain patients: relationship to patient characteristics and current adjustment. Pain 1983;17:33-44. [PubMed: 6226916]

8••. Sullivan MJL, Bishop SR, Pivik J. The pain catastrophizing scale: development and validation. Psychol Assess 1995;7(4):524-532.Concerns the construction and validation of the Pain Catastrophizing Scale (PCS), the most comprehensive assessment instrument to date of the catastrophizing construct. The authors demonstrate a three-factor hierarchical structure for the PCS, including magnification, helplessness and rumination dimensions

9. Osman A, Barrios FX, Kopper BA, et al. Factor structure, reliability, and validity of the pain catastrophizing scale. J Behav Med 1997;20(6):589-605. [PubMed: 9429990]

10. Osman A, Barrios FX, Gutierrez PM, et al. The pain catastrophizing scale: further psychometric evaluation with adult samples. J Behav Med 2000;23(4):351-365. [PubMed: 10984864]

11. Tremblay I, Beaulieu Y, Bernier A, et al. Pain catastrophizing scale for francophone adolescents: a preliminary validation. Pain Res Manag 2008;13(1):19-24. [PubMed: 18301812]

12. Lu Q, Tsao JCI, Myers CD, et al. Coping Predictors of children's laboratory-induced pain tolerance, intensity, and unpleasantness. J Pain 2007;8(9):708-717. [PubMed: 17611165]

13. Granot M, Ferber SG. The roles of pain catastrophizing and anxiety in the prediction of postoperative pain intensity. Clin J Pain 2005;21(5):439-445. [PubMed: 16093750]

14. Meyer K, Sprott H, Mannion AF. Cross-cultural adaptation, reliability, and validity of the German version of the Pain Catastrophizing Scale. J Psychosomatic Res 2008;64(5):469-478.

15. Yap JC, Lau J, Chen PP, et al. Validation of the Chinese Pain Catastrophizing Scale (HK-PCS) in patients with chronic pain. Pain Med 2008;9(2):186-195. [PubMed: 18298701]

16. Miro J, Nieto R, Huguet A. The Catalan version of the Pain Catastrophizing Scale: a useful instrument to assess catastrophic thinking in whiplash patients. J Pain 2008;9(5):397-406. [PubMed: 18226964]

17. Chibnall JT, Tait RC. Confirmatory factor analysis of the Pain Catastrophizing Scale in African American and Caucasian Workers' Compensation claimants with low back injuries. Pain 2005;113 (3):369-375. [PubMed: 15661446]

18. D'Eon JL, Harris CA, Ellis JA. Testing factorial validity and gender invariance of the pain catastrophizing scale. J Behav Med 2004;27(4):361-372. [PubMed: 15559733]

19. Dixon KE, Thorn BE, Ward LC. An evaluation of sex differences in psychological and physiological responses to experimentally-induced pain: a path analytic description. Pain 2004;112:188-196. [PubMed: 15494200]

20. Edwards RR, Campbell CM, Fillingim RB. Catastrophizing and experimental pain sensitivity: only in vivo reports of catastrophic cognitions correlate with pain responses. J Pain 2005;6(5):338-339. [PubMed: 15890636]

21. Edwards RR, Smith MT, Stonerock G, Haythornthwaite JA. Pain-related catastrophizing in healthy women is associated with greater temporal summation of an reduced habituation to thermal pain. Clin J Pain 2006;22:730-737. [PubMed: 16988570]

22. Hirsh AT, George SZ, Bialosky JE, Robinson ME. Fear of pain, pain catastrophizing, and acute pain perception: relative prediction and timing of assessment. J Pain 2008;9(9):806-812. [PubMed: 18486557]

23. Edwards RR, Kronfli T, Haythornthwaite JA, et al. Association of catastrophizing with interleukin-6 responses to acute pain. Pain 2008;140(1):135-144. [PubMed: 18778895]

24. Sullivan MJ, D'Eon JL. Relation between catastrophizing and depression in chronic pain patients. J Abnorm Psychol 1990;99(3):260-263. [PubMed: 2145334] 
25. Affleck G, Tennen H, Urrows S, Higgins P. Neuroticism and the pain-mood relation in rheumatoid arthritis: insights from a prospective daily study. J Consult Clin Psychol 1992;60(1):119-126. [PubMed: 1556274]

26. Hirsh AT, George SZ, Riley JL III, Robinson ME. An evaluation of the measurement of pain catastrophizing by the coping strategies questionnaire. Eur J Pain 2007;11(1):75-81. [PubMed: 16545973]

27. Hirsh A, George S, Riley J, Robinson M. Sex differences and construct redundancy of the Coping Strategies Questionnaire - catastrophizing Subscale. J Pain 2005;6(3 Suppl 1):S60.

28•. George SZ, Dannecker EA, Robinson ME. Fear of pain, not pain catastrophizing, predicts acute pain intensity, but neither factor predicts tolerance or blood pressure reactivity: an experimental investigation in pain-free individuals. Eur J Pain 2006;10(5):457-465. [PubMed: 16095935]As we underscore throughout the review, it is of importance to examine unique versus common contributions of pain catasrophizing and conceptually related variables to pain-related outcomes. This interesting manuscript shows that pain-related fear accounts for unique variance in acute pain intensity ratings, but not pain catastrophizing when both are simultaneously considered in analyses.

29. Sullivan MJL, Thorn B, Rodgers W, Ward C. Path model of antecdents to pain experience: experimental and clinical findings. Clin J Pain 2004;20(3):164-173. [PubMed: 15100592]

30• Sullivan MJ, Thorn B, Haythornthwaite JA, et al. Theoretical perspectives on the relation between pain catastrophizing and pain. Clin J Pain 2001;17:52-64. [PubMed: 11289089]Seminal review for the pain catastrophizing literature. The current article presents data that have been accumulated based on notions first espoused in this article, as well as presenting novel ideas and findings.

31. Edwards RR, Bingham CO III, Bathon J, Haythornthwaite JA. Catastrophizing and pain in arthritis, fibromyalgia and other rheumatic disease. Arthritis Rheum 2006;55:325-332. [PubMed: 16583384]

32. Keefe FJ, Rumble ME, Scipio CD, et al. Psychological aspects of persistent pain: current state of the science. J Pain 2004;5(4):195-211. [PubMed: 15162342]

33. Haythornthwaite JA, Clark MR, Pappagallo M, Raja SN. Pain coping strategies play a role in the persistence of pain in post-herpetic neuralgia. Pain 2003;106(3):453-460. [PubMed: 14659529]

34. Keefe FJ, Caldwell DS, Martinez S, et al. Analyzing pain in rheumatoid arthritis patients. Pain coping strategies in patients who have had knee replacement surgery. Pain 1991;46(2):153-160. [PubMed: 1749638]

35. Richardson C, Glenn S, Horgan M, Nurmikko T. A prospective study of factors associated with the presence of phantom limb pain six months after major lower limb amputation in patients with peripheral vascular disease. J Pain 2007;8(10):793-801. [PubMed: 17631056]

36. Kendall K, Saxby B, Farrow M, Naisby C. Psychological factors associated with short-term recovery from total knee replacement. Br J Health Psychol 2001;6(1):41-52. [PubMed: 14596737]

37. Roth ML, Tripp DA, Harrison MH, Sullivan M, Carson P. Demographic and psychosocial predictors of acute perioperative pain for total knee arthroplasty. Pain Res Manag 2007;12(3):184-194.

38. Forsythe ME, Dunbar MJ, Hennigar AW, Sullivan MJ, Gross M. Prospective relation between catastrophizing and residual pain following knee arthroplasty: two-year follow-up. Pain Res Manag 2008;13(4):335-441. [PubMed: 18719716]

39. van Wijk RMAW, Geurts JWM, Lousberg R, et al. Psychological predictors of substantial pain reduction after minimally invasive radiofrequency and injection treatments for chronic low back pain. Pain Med 2008;9(2):212-221. [PubMed: 18298704]

40. Samwel H, Slappendel R, Crul BJP, Voerman VF. Psychological predictors of the effectiveness of radiofrequency lesioning of the cervical spinal dorsal ganglion (RF-DRG). Eur J Pain 2000;4(2): 149-155. [PubMed: 10957696]

41. Groth-Marnat G, Fletcher A. Influence of neuroticism, catastrophizing, pain duration, and receipt of compensation on short-term response to nerve block treatment for chronic back pain. J Behav Med 2000;23(4):339-350. [PubMed: 10984863]

42. Tang NK, Crane C. Suicidality in chronic pain: a review of the prevalence, risk factors and psychological links. Psychol Med 2006;36(5):575-586. [PubMed: 16420727]

43. Edwards RR, Smith MT, Kudel I, Haythornthwaite J. Pain-related catastrophizing as a risk factor for suicidal ideation in chronic pain. Pain 2006;126(1-3):272-279. [PubMed: 16926068]

44. Lazarus, R.; Folkman, S. Stress, Appraisal and Coping. Springer; NY, USA: 1984. 
45. Severeijns R, Vlaylen JWS, van den Hout MA. Do we need a communal coping model of pain catastrophizing? An alternative explanation. Pain 2004;111:226-229. [PubMed: 15363864]

46. Eccleston C, Crombez G. Pain demands attention: a cognitive-affective model of the interruptive function of pain. Psychol Bull 1999;125(3):356-366. [PubMed: 10349356]

47. Crombez G, Vlaeyen JW, Heuts PH, Lysens R. Pain-related fear is more disabling than pain itself: evidence on the role of pain-related fear in chronic back pain disability. Pain 1999;80(1-2):329-339. [PubMed: 10204746]

48. Van Damme S, Crombez G, Eccleston C. Retarded disengagement from pain cues: the effects of pain catastrophizing and pain expectancy. Pain 2002;100(1-2):111-118. [PubMed: 12435464]

49•. Van Damme S, Crombez G, Eccleston C. Disengagement from pain: the role of catastrophic thinking about pain. Pain 2004;107(1-2):70-76. [PubMed: 14715391]Presents a wonderful and creative manner by which to examine attention bias/information processing processes underlying pain catastrophizing. Using a cueing paradigm, the authors found that low and high pain catastrophizers do not differentially engage their attention resources to pain-related information. However, high pain catastrophizers showed pronounced difficulty disengaging their attention focus from painrelated information relative to low catastrophizers.

50. Goubert L, Francken G, Crombez G, Vansteenwegen D, Lysens R. Exposure to physical movement in chronic back pain patients: no evidence for generalization across different movements. Behav Res Ther 2002;40(4):415-429. [PubMed: 12002898]

51. Asmundson GJ, Kuperos JL, Norton GR. Do patients with chronic pain selectively attend to painrelated information? Preliminary evidence for the mediating role of fear. Pain 1997;72(1-2):27-32. [PubMed: 9272784]

52. Asmundson GJ, Hadjistavropoulos HD. Is high fear of pain associated with attentional biases for pain-related or general threat? A categorical reanalysis. J Pain 2007;8(1):11-18. [PubMed: 17207740]

53. Vancleef LMG, Peters ML. Pain catastrophizing, but not injury/illness sensitivity or anxiety sensitivity, enhances attentional interference by pain. J Pain 2006;7(1):23-30. [PubMed: 16414550]

54. Vangronsveld K, Damme SV, Peters M, et al. An experimental investigation on attentional interference by threatening fixations of the neck in patients with chronic whiplash syndrome. Pain 2007;127(1-2):121-128. [PubMed: 16971044]

55. Goubert L, Crombez G, Van DS. The role of neuroticism, pain catastrophizing and pain-related fear in vigilance to pain: a structural equations approach. Pain 2004;107:234-241. [PubMed: 14736586]

56. Michael ES, Burns JW. Catastrophizing and pain sensitivity among chronic pain patients: moderating effects of sensory and affect focus. Annals Behav Med 2004;27(3):185-194.

57. Keefe FJ, Lipkus I, Lefebvre JC, et al. The social context of gastrointestinal cancer pain: a preliminary study examining the relation of patient pain catastrophizing to patient perceptions of social support and caregiver stress and negative responses. Pain 2003;103(1-2):151-156. [PubMed: 12749969]

58. Giardino ND, Jensen MP, Turner JA, et al. Social environment moderates the association between catastrophizing and pain among persons with a spinal cord injury. Pain 2003;106(1-2):19-25. [PubMed: 14581106]

59. Sullivan MJL, Martel MO, Tripp D, et al. The relation between catastrophizing and the communication of pain experience. Pain 2006;122(3):282-288. [PubMed: 16545907]

60. Boothby JL, Thorn BE, Overduin LY, Ward CL. Catastrophizing and perceived partner responses to pain. Pain 2004;109(3):500-506. [PubMed: 15157712]

61. Buenaver LF, Edwards RR, Haythornthwaite JA. Pain-related catastrophizing and perceived social responses: inter-relationships in the context of chronic pain. Pain 2007;127(3):234-242. [PubMed: 17011706]

62. Cano A. Pain catastrophizing and social support in married individuals with chronic pain: the moderating role of pain duration. Pain 2004;110(3):656-664. [PubMed: 15288406]

63. Lackner JM, Gurtman MB. Pain catastrophizing and interpersonal problems: a circumplex analysis of the communal coping model. Pain 2004;110(3):597-604. [PubMed: 15288400]Employed concepts from interpersonal theory (i.e., an interpersonal circumplex model) by which to examine the interpersonal characteristics and correlates of pain catastrophizing. They found that catastrophizers were characterized by andinterpersonal style demanding support and care-taking. 
This article, by integrating concepts from existing and well-validated theory and methodology, advanced our knowledge of the interpersonal correlates of pain catastrophizing.

64. McWilliams LA, Asmundson GJG. The relationship of adult attachment dimensions to pain-related fear, hypervigilance, and catastrophizing. Pain 2007;127(1-2):27-34. [PubMed: 16963183]

65. France CR, France JL, al'Absi M, et al. Catastrophizing is related to pain ratings, but not nociceptive flexion reflex threshold. Pain 2002;99(3):459-463. [PubMed: 12406521]

66. Weissman-Fogel I, Sprecher E, Pud D. Effects of catastrophizing on pain perception and pain modulation. Exp Brain Res 2008;186(1):79-85. [PubMed: 18030455]

67. Eide PK. Wind-up and the NMDA receptor complex from a clinical perspective. Eur J Pain 2000;4 (1):5-15. [PubMed: 10833550]

68. Edwards RR, Fillingim RB, Maixner W, et al. Catastrophizing predicts changes in thermal pain responses after resolution of acute dental pain. J Pain 2004;5(3):164-170. [PubMed: 15106129]

69. Granot M, Granovsky Y, Sprecher E, et al. Contact heat-evoked temporal summation: tonic versus repetitive-phasic stimulation. Pain 2006;122(3):295-305. [PubMed: 16540248]

70. George SZ, Wittmer VT, Fillingim RB, Robinson ME. Sex and pain-related psychological variables are associated with thermal pain sensitivity for patients with chronic low back pain. J Pain 2007;8 (1):2-10. [PubMed: 17207739]

71. Quartana P, Burns J, Lofland K. Attentional strategy moderates effects of pain catastrophizing on symptom-specific physiological responses in chronic low back pain patients. J Behav Med 2007;30 (3):221-231. [PubMed: 17443402]

72. Wolff B, Burns J, Quartana P, et al. Pain catastrophizing, physiological indexes, and chronic pain severity: tests of mediation and moderation models. J Behav Med 2008;31(2):105-114. [PubMed: 18158618]

73. Flor H, Turk DC. Psychophysiology of chronic pain: do chronic pain patients exhibit symptomspecific psychophysiological responses? Psychol Bull 1989;105(2):215-259. [PubMed: 2648442]

74. Flor H, Birbaumer N, Schugens MM, Lutzenberger W. Symptom-specific psychophysiological responses in chronic pain patients. Psychophysiology 1992;29(4):452-460. [PubMed: 1410176]

75. Johansson AC, Gunnarsson LG, Linton SJ, et al. Pain, disability and coping reflected in the diurnal cortisol variability in patients scheduled for lumbar disc surgery. Eur J Pain 2008;12(5):633-640. [PubMed: 18077197]

76. Edwards RR, Kronfli T, Haythornthwaite JA, et al. Association of catastrophizing with interleukin-6 responses to acute pain. Pain 2008;140(1):135-144. [PubMed: 18778895]

77. Diatschenko L, Slade GD, Nackley AG, et al. Genetic basis for individual variations in pain perception and the development of a chronic pain condition. Mol Genetics 2005;14:134-143.

78. Diatschenko L, Nackley AG, Slade GD, et al. Catechol- $O$-methyltransferase gene polymorphisms are associated with multiple pain-evoking stimuli. Pain 2006;125:216-224. [PubMed: 16837133]

79• . George SZ, Wallace MR, Wright TW, et al. Evidence for a biopsychosocial influence on shoulder pain: pain catastrophizing and catechol- $O$-methyltransferase (COMT) diplotype predict clinical pain ratings . Pain 2008;136(1-2):53-61. [PubMed: 17686583]Explored the interaction of pain catastrophizing and a high pain sensitivity genetic diplotype to predict pain ratings in shoulder pain patients. This study nicely reveals that pain catastrophizing's effects on pain-related outcomes may be moderated by genetic factors

80. George SZ, Dover GC, Wallace MR, et al. Biopsychosocial influence on exercise-induced delayed onset muscle soreness at the shoulder: pain catastrophizing and catchol- $O$-methyltransferase (COMT) diplotype predict pain. Clin J Pain 2008;24(9):793-801. [PubMed: 18936597]

81•• Tracey I. Imaging pain. Br J Anaesth 2008;101(1):32-39. [PubMed: 18556697]A comprehensive and thoughtful overview of novel imaging technologies and approaches for furthering our understanding of pain.

82. Rainville P. Brain mechanisms of pain affect and pain modulation. Curr Opin Neurobiol 2002;12(2): 195-204. [PubMed: 12015237]

83. Wager TD, Davidson ML, Hughes BL, Lindquist MA, Ochsner KN. Prefrontal-subcortical pathways mediating successful emotion regulation. Neuron 2008;59(6):1037-1050. [PubMed: 18817740]

84. Seminowicz DA, Davis KD. Cortical responses to pain in healthy individuals depends on pain catastrophizing. Pain 2006;120(3):297-306. [PubMed: 16427738] 
85. Ochsner KN, Ludlow DH, Knierim K, et al. Neural correlates of individual differences in pain-related fear and anxiety. Pain 2006;120(1-2):69-77. [PubMed: 16364548]

86•. Gracely RH, Geisser ME, Giesecke T, et al. Pain catastrophizing and neural responses to pain among persons with fibromyalgia. Brain 2004;127(4):835-843. [PubMed: 14960499]Provides evidence that pain catastrophizing is associated with augmented brain activation during pain stimulation in areas related to, in particular, the modulation and experience of affective aspects of the pain experience. Using imaging technology, we can further ourunderstanding of brain-behavior relations in the context of pain catastrophizing.

87. Turk DC. Cognitive-behavioral approach to the treatment of chronic pain patients. Reg Anesth Pain Med 2003;28(6):573-579. [PubMed: 14634950]

88. Burns JW, Johnson BJ, Mahoney N, et al. Cognitive and physical capacity process variables predict long-term outcome after treatment of chronic pain. J Consult Clin Psychol 1998;66(2):434-439. [PubMed: 9583347]

89. Jensen MP, McFarland CA. Increasing the reliability and validity of pain intensity measurement in chronic pain patients. Pain 1993;55:195-203. [PubMed: 8309709]

90. Jensen MP, Turner JA, Romano JM, Lawler BK. Relationship of pain-specific beliefs to chronic pain adjustment. Pain 1994;55:301-309. [PubMed: 7936708]

91. Jensen MP, Turner JA, Romano JM. Changes in beliefs, catastrophizing, and coping are associated with improvement in multidisciplinary pain treatment. J Consult Clin Psychol 2001;69(4):655-662. [PubMed: 11550731]

92. Jensen MP, Turner JA, Romano JM. Changes after multidisciplinary pain treatment in patient pain beliefs and coping are associated with concurrent changes in patient functioning. Pain 2007;131(12):38-47. [PubMed: 17250963]

93. Finkel, SE. Causal Analysis of Panel Data. Sage University Paper Series on Quantitative Applications in the Social Sciences. Sage Publications; Beverly Hills, CA, USA: 1995.

94. Burns JW, Kubilus A, Bruehl S, et al. Do changes in cognitive factors influence outcome following multidisciplinary treatment for chronic pain? A cross-lagged panel analysis. J Consul Clin Psychol 2003;71(1):81-91.

95••. Burns JW, Glenn B, Bruehl S, et al. Cognitive factors influence outcome following multidisciplinary chronic pain treatment: a replication and extension of a cross-lagged panel analysis. Behav Res Ther 2003;41(10):1163-1182. [PubMed: 12971938]Represents a comprehensive analytical approach to the temporal precedence of pain catastrophizing in the context of the pain treatment.

96. Turner JA, Mancl L, Aaron LA. Brief cognitive-behavioral therapy for temporomandibular disorder pain: effects on daily electronic outcome and process measures. Pain 2005;117(3):377-387. [PubMed: 16153777]

97. Turner JA, Mancl L, Aaron LA. Short- and long-term efficacy of brief cognitive-behavioral therapy for patients with chronic temporomandibular disorder pain: a randomized, controlled trial. Pain 2006;121(3):181-194. [PubMed: 16495014]

98. Turner JA, Holtzman S, Mancl L. Mediators, moderators, and predictors of therapeutic change in cognitive-behavioral therapy for chronic pain. Pain 2007;127(3):276-286. [PubMed: 17071000]

99. George SZ, Zeppieri J, Cere AL, et al. A randomized trial of behavioral physical therapy interventions for acute and sub-acute low back pain (NCT00373867). Pain 2008;140(1):145-157. [PubMed: 18786762]

100••. Smeets RJ, Vlaeyen JW, Kester AD, Knottnerus JA. Reduction of pain catastrophizing mediates the outcome of both physical and cognitive-behavioral treatment in chronic low back pain. J Pain 2006;7(4):261-271. [PubMed: 16618470]Provides evidence that reductions in pain catastrophizing mediate pain-related outcomes for cognitive-behavioral as well as physical rehabilitation interventions. Studies of this sort have potential to shed sufficient versus necessary treatment factors required to change pain catastrophizing

101. Dobson KS, Hollon SD, Dimidjian S, et al. Randomized trial of behavioral activation, cognitive therapy, and antidepressant medication in the prevention of relapse and recurrence in major depression. J Consult Clin Psychol 2008;76(3):468-477. [PubMed: 18540740] 
102. Burns JW, Glenn B, Lofland K, Bruehl S, Harden RN. Stages of change in readiness to adopt a selfmanagement approach to chronic pain: the moderating role of early-treatment stage progression in predicting outcome. Pain 2005;115(3):322-331. [PubMed: 15911159]

103. Glenn B, Burns JW. Pain self-management in the process and outcome of multidisciplinary treatment of chronic pain: evaluation of a stage of change model. J Behav Med 2003;26(5):417-433. [PubMed: 14593851]

104. Strand EB, Kerns RD, Christie A, Haavik-Nilsen K, Klokkerud M, Finset A. Higher levels of pain readiness to change and more positive affect reduce pain reports - a weekly assessment study on arthritis patients. Pain 2007;127(3):204-213. [PubMed: 16997472]

105. Lackner JM, Quigley BM. Pain catastrophizing mediates the relationship between worry and pain suffering in patients with irritable bowel syndrome. Behav Res Ther 2005;43(7):943-957. [PubMed: 15896288]

106. Severeijns R, van den Hout MA, Vlaeyen JW. The causal status of pain catastrophizing: an experimental test with healthy participants. Eur J Pain 2005;9(3):257-265. [PubMed: 15862475]

107. MacLeod C, Rutherford E, Campbell L, Ebsworthy G, Holker L. Selective attention and emotional vulnerability: assessing the causal basis of their association through the experimental manipulation of attentional bias. J Abnorm Psychol 2002;111(1):107-123. [PubMed: 11866165]

108. Amir N, Weber G, Beard C, Bomyea J, Taylor CT. The effect of a single-session attention modification program on response to a public-speaking challenge in socially anxious individuals. J Abnorm Psychol 2008;117(4):860-868. [PubMed: 19025232]

109. Amir N, Beard C, Burns M, Bomyea J. Attention modification program in individuals with generalized anxiety disorder. J Abnorm Psychol 2009;118(1):28-33. [PubMed: 19222311]

110• Goubert L, Vervoort T, Cano A, Crombez G. Catastrophizing about their children's pain is related to higher parent-child congruency in pain ratings: an experimental investigation. Eur J Pain 2009;13 (2):196-201. [PubMed: 18448370]A wonderful attempt to examine the influence of a child's level of pain catastrophizing on parental report of children's pain. Studies such as this may have implications for long-term maintenance of pain catastophizing via parent-child interactions. 


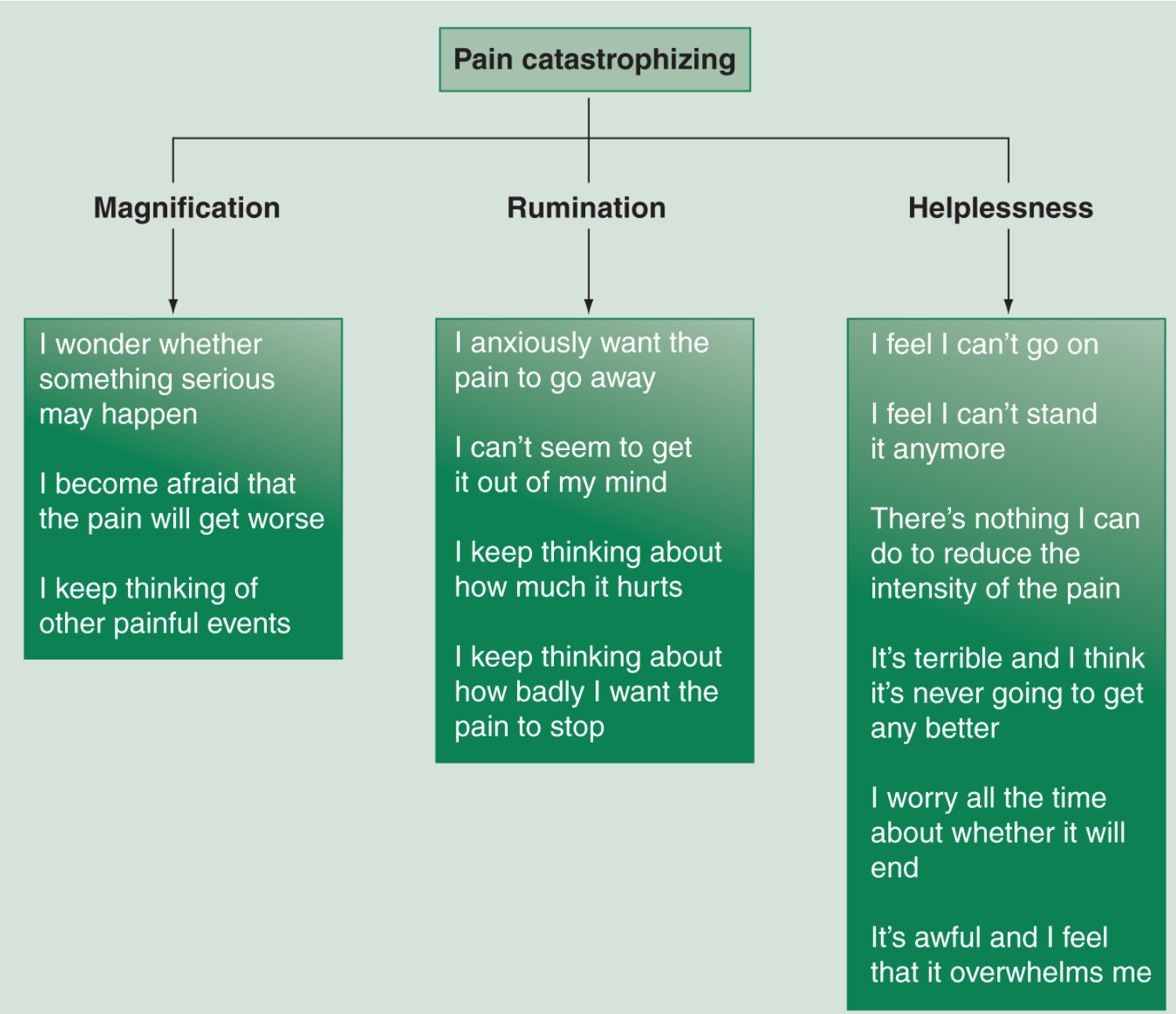

Figure 1.

Hierarchical factor structure of pain catastrophizing and corresponding Pain Catastrophizing Scale items. 


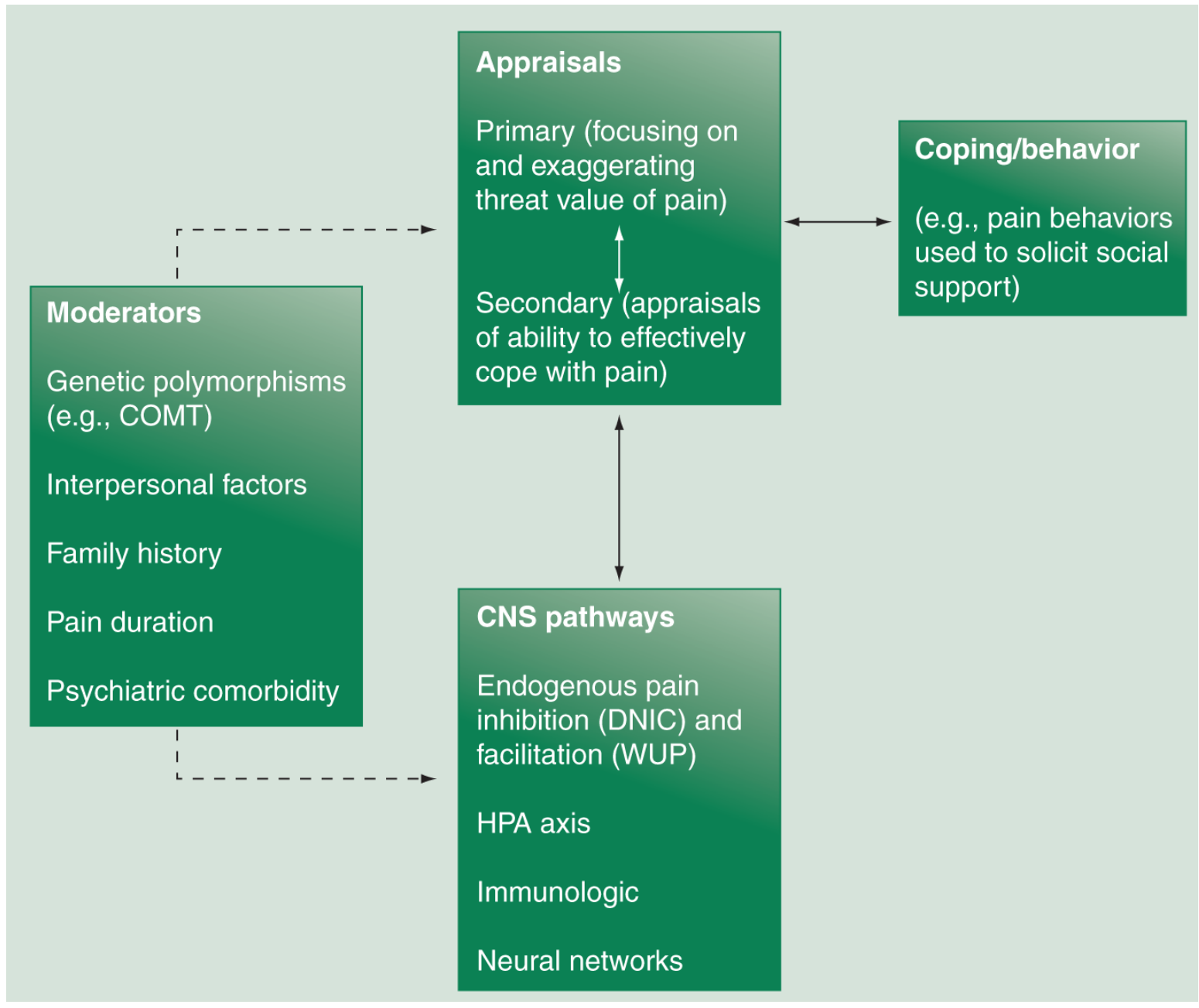

Figure 2. A proposed multiprocess framework for pain catastrophizing research

COMT: Catchol- $O$-methyltransferase; DNIC: Diffuse noxious inhibitory controls. 
Table 1

Theoretical conceptualizations of pain catastrophizing.

\begin{tabular}{|c|c|}
\hline Conceptualization & Details \\
\hline Appraisal theory & $\begin{array}{l}\text { Pain catastrophizing is viewed as an appraisal process. Painful stimuli are appraised in a primary } \\
\text { (magnification, rumination) and secondary (helplessness) fashion. Behavior is deployed based on this } \\
\text { appraisal process }\end{array}$ \\
\hline Attention bias/information processing & $\begin{array}{l}\text { Pain catastrophizing is comprised of an information processing or attention bias to pain-related stimuli, } \\
\text { particularly the negative affective or 'hot' dimensions of such stimuli }\end{array}$ \\
\hline Communal coping & $\begin{array}{l}\text { Pain catastrophizing is an interpersonal coping style that is used to solicit interpersonal support of a } \\
\text { tangible and emotional nature }\end{array}$ \\
\hline CNS mechanisms & $\begin{array}{l}\text { Pain catastrophizing is associated with altered CNS processes, such as enhanced temporal summation, } \\
\text { aberrant hypothalamic-pituitary-adrenal axis activity and altered cytokine responses to pain }\end{array}$ \\
\hline Neural underpinnings & $\begin{array}{l}\text { Pain catastrophizing is associated with activation in brain regions implicated in processing of affective } \\
\text { dimensions of pain; regions that are also implicated in the cognitive regulation of emotion and cognition } \\
\text { (e.g., anterior cingulate cortex and ventromedial and dorsolateral prefrontal cortices) }\end{array}$ \\
\hline
\end{tabular}

